\title{
Elucidation of the Molecular Interaction between miRNAs and the HOXA9 Gene, Involved in Acute Myeloid Leukemia, by the Assistance of Argonaute Protein through a Computational Approach
}

\author{
Rohit Pritam Das ${ }^{1}$, V. Badireenath Konkimalla ${ }^{2}$, Surya Narayan Rath ${ }^{1 *}$, \\ Jagadish Hansa ${ }^{3}$, Manaswini Jagdeb ${ }^{1}$ \\ ${ }^{1}$ BIF Centre, Department of Bioinformatics, Orissa University of Agriculture \& Technology, Bhubaneswar 751003, India, \\ ${ }^{2}$ School of Biological Sciences, National Institute of Science Education and Research, Bhubaneswar 751005, India, \\ ${ }^{3}$ Department of Surgical Oncology, IMS and SUM Hospital, SOA University, Bhubaneswar 751003, India
}

\begin{abstract}
Acute myeloid leukemia is a well characterized blood cancer in which the unnatural growth of immature white blood cell takes place, where several genes transcription is regulated by the micro RNAs (miRNAs). Argonaute (AGO) protein is a protein family that binds to the miRNAs and mRNA complex where a strong binding affinity is crucial for its RNA silencing function. By understanding pattern recognition between the miRNAs-mRNA complex and its binding affinity with AGO protein, one can decipher the regulation of a particular gene and develop suitable siRNA for the same in disease condition. In the current work, HOXA9 gene has been selected from literature, whose deregulation is well-established in acute myeloid leukemia. Four miRNAs (mir-145, mir-126, let-7a, and mir-196b) have been selected to target mRNA of HOXA9 (NCBI accession No. NM_152739.3). The binding interaction between mRNAs and mRNA of HOXA9 gene was studied computationally. From result, it was observed mir-145 has highest affinity for HOXA9 gene. Furthermore, the interaction between miRNAs-mRNA duplex of all chosen miRNAs are docked with AGO protein (PDB ID: 3F73, chain A) to study their interaction at molecular level through an in silico approach. The residual interaction and hydrogen bonding are inspected in Discovery Studio 3.5 suites. The current investigation throws light on understanding of AGO-assisted miRNA based gene silencing mechanism in HOXA9 gene associated in acute myeloid leukemia computationally.
\end{abstract}

Keywords: acute myeloid leukemia, Argonaute, HOXA9, in silico, microRNAs, mRNA

\section{Introduction}

MicroRNAs (miRNAs) are small non-coding single stranded RNAs ( $18-22 \mathrm{bp})$ found in most eukaryotes, playing a crucial role in a wide range of biological processes, such as host-viral interaction, apoptosis, stem cell maintenance, and metabolism, etc. miRNAs are involved in gene silencing mechanisms by targeting un-translated regions of mRNA through sequence complementarity $[1,2]$, and approximately $30 \%$ of protein-coding genes are regulated by miRNAs [3]. As many genes can be regulated by a single or multiple miRNAs, an immense interest has been created to understand
miRNA-based gene regulation through the identification of miRNAs, as well as their target genes. The Argonaute (AGO) class of proteins plays a crucial functional role in the molecular recognition of miRNA-mRNA hybrids during the process of gene regulation $[4,5]$, and mutations in either the sequence of miRNAs or the miRNA target sites have a significant contribution in the manifestation of cancer due to the loss of gene regulation $[5,6]$.

Leukemia is a cancer of white blood cells that is commonly classified as acute myeloid leukemia (AML), acute lymphoblastic leukemia, and chronic lymphoblastic leukemia. AML is a well-characterized blood cancer in which the unnatural growth of immature white blood cells takes place, 
where several genes involved are either up-regulated or down regulated by miRNAs. Though the importance of miRNAs in gene regulation $[2-5,7,8]$ and their regulation by RNA-induced silencing complexes [7] are well established, the mechanism of miRNA-induced gene silencing at the molecular level is still unclear [9, 10]. Despite several detailed research studies, controlling abnormal cell proliferation at the molecular level through miRNA-mediated gene silencing is still a major challenge. Therefore, with the current knowledge, attempts have been made to study the AGO protein-assisted molecular interaction between miRNAs and their target genes, and a computational approach [11] in this regard will be of great aid and value. Here, in this study, the HOXA9 gene was selected from the literature as an important target in AML, and in silico approaches, like miRNA target site prediction, miRNA-mRNA duplex prediction, and molecular docking between AGO protein and four miRNAs, such as mir-145, mir-126, let7a, and mir-196b $[8,12,13]$, were conducted to elucidate the AGO protein-assisted molecular interaction between miRNAs and the HOXA9 gene in association with AML [14, 15] computationally.

\section{Methods}

\section{Selection of HOXA9 gene as a target in AML}

The leukemia gene database (http://www.bioinformatics. org/legend/leuk_db.htm) documented the AF10, NUP98, CBFB, NUP214, HOXA9, CREBBP, ARHGEF12, CDX2, LCP1, CEBPA, DEK, FUS, and RUNX1 genes to be involved in AML. Among them, the HOXA9 gene is reported to have major relevance to their regulatory functions and aberrant expression in both mice and humans $[14,15]$. Furthermore, identification of miRNAs that target the HOXA9 gene would help us to understand its interaction at the molecular level in AML conditions.

\section{Selection of miRNAs targeting the HOXA9 gene}

Firstly, from the literature, the miRNAs with affinity for the HOXA9 gene were identified $[12,13]$, which was further verified by prediction of miRNA target sites in the HOXA9 gene using the miRTarBase web server (http://mirtarbase. mbc.nctu.edu.tw/). The search showed four miRNAs - namely, mir-145, mir-126, let-7a, and mir-196b - that have target sites within HOXA9 (Supplementary Tables 1 and 2). Two different binding sites were identified for mir-196b in the HOXA9 gene, denoted mir-196b.1 and mir-196b.2, respectively.

\section{Prediction of duplex between miRNAs and mRNA of the HOXA9 gene}

A computational approach was carried out to study the binding mode of selected miRNAs (mir-145, mir-126, let-7a, mir-196b.1, and mir-196b.2) with the mRNA of HOXA9 to confirm the selection of the best miRNA. The properties, like predicted binding sites of HOXA9 for miRNAs (Supplementary Table 3) and minimum free energy (MFE) score of duplex interactions, were used for confirmation of binding. Two different duplexes were formed for mir-196b (represented as mir-196b.1 and mir-196b.2) with HOXA9 due to the identification of two target sites in HOXA9 (Supplementary Table 3). The MFE scores for folding of miRNAs and the mRNA duplex (Supplementary Table 4) were calculated using the mfold web server (http://mfold.rna.albany.edu/? q=mfold). The appropriate secondary structures of miRNAs and the mRNA duplex (Supplementary Table 4) were predicted using the Centroid Fold web server (http://www.ncrna.org/ centroidfold/). The prediction of tertiary structures of the duplex were performed in 3dRNA web server (http://biophy. hust.edu.cn/3dRNA/3dRNA.html) using dot bracket structures (Supplementary Table 4) that resulted from the Centroid Fold.

\section{Molecular interaction between miRNAs and AGO protein}

To illustrate the assistance of catalytic AGO protein for the binding of miRNAs (mir-145, mir-126, let-7a, mir-196b.1, and mir-196b.2) with the HOXA9 gene, docking between AGO protein and miRNA-mRNA duplexes was performed in the PachDock online server [16]. The PachDock algorithm ranked the docked complexes on the basis of geometrical shape complementary scores. Close amino acid residue interactions and participating atoms in hydrogen bonding were inspected and analyzed in the Discovery Studio 3.5 suite (http://accelrys.com/products/discovery-studio/visualization- download.php).

\section{Results}

\section{Duplex interaction between miRNAs and mRNA of the HOXA9 gene}

The leukemia gene database search result, supported by literature evidence $[14,15]$, signifies that HOXA9 (NCBI accession No. NM_152739.3) has aberrant expression at the molecular level and was therefore selected as a target gene in AML. Four miRNAs - namely, mir-145, mir-126, let-7a, and mir-196b (Supplementary Table 2) - were taken from the literature, and their target sites in the HOXA9 gene were verified through the miRTarBase web server. The predicted binding regions in the HOXA9 gene (Supplementary Tables 1 and 3) for the miRNAs above were supported by good MFE scores calculated for the miRNAs and mRNA duplex (Supplementary Table 4) using the mfold web server, which 


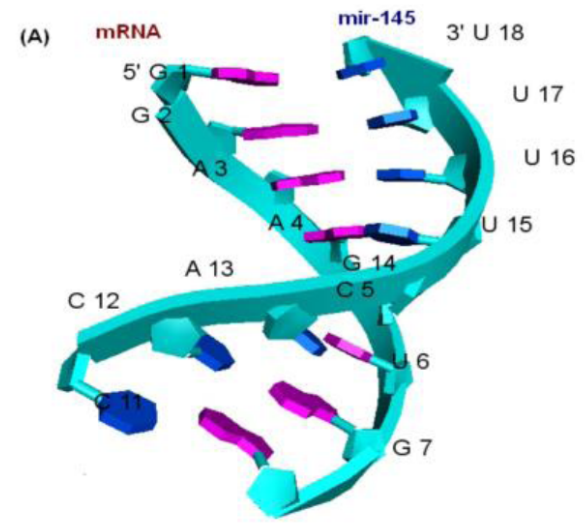

G 8

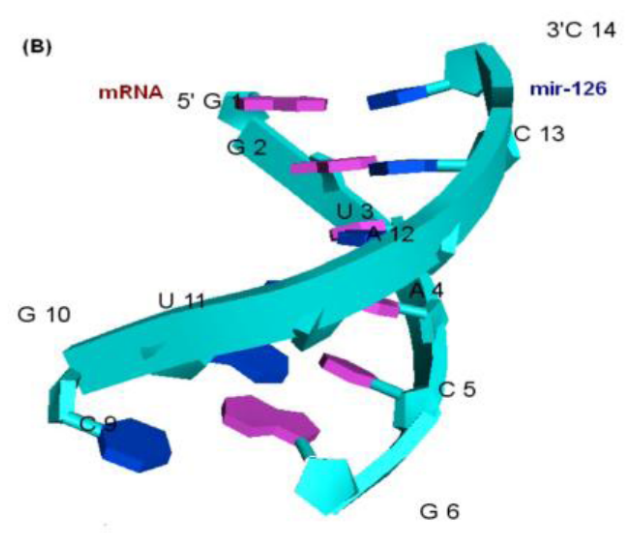

(D)

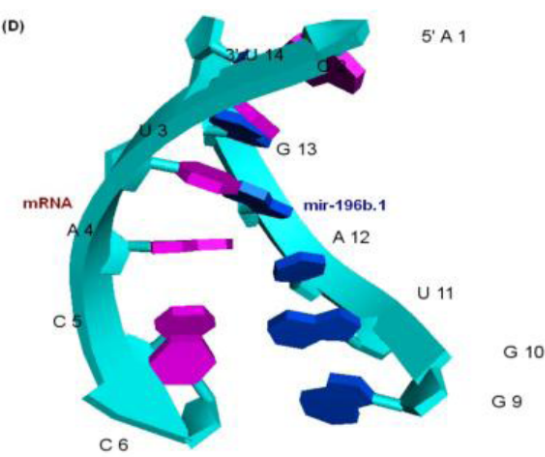

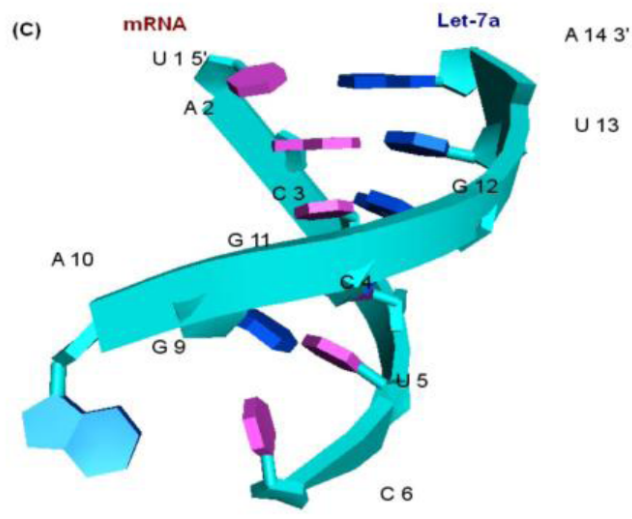

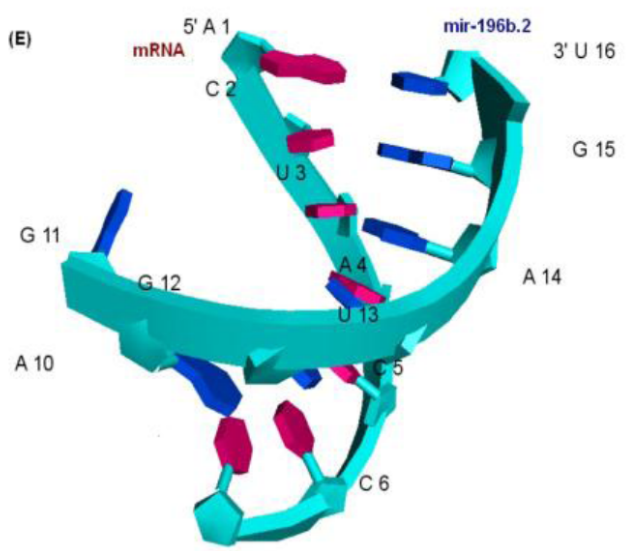

Fig. 1. Three dimensional binding modes of mir-145, mir-126, let-7a, mir-196b.1, and mir-196b.2 with mRNA of HOXA9 gene are deciphered in section $(A),(B),(C),(D)$, and $(E)$, respectively. 
implicates a strong affinity between them at the molecular level. Three-dimensional binding modes of all miRNAs and mRNA duplexes (mir-145, mir-126, let-7a, mir-196b.1, and mir-196b.2) were constructed and deciphered (Fig. 1A-1E) using the Discovery Studio 3.5 suite.

\section{Study of binding affinity between miRNAs and AGO protein through docking}

The three-dimensional structure of AGO protein, with 685 amino acid residues, was retrieved from Protein Data Bank (PDB ID: 3F73, chain A). The AGO protein is a silencing complex structure with a seed containing guide DNA and target RNA duplexes. The structure preparation and correction of AGO protein were performed in Discovery Studio 3.5 suite before docking. The docking between miRNA-mRNA duplexes (mir-145, mir-126, let-7a, mir-196b.1, and mir-196b.2) and AGO protein was carried out separately in the PachDock online server on the basis of geometrical shape complementary scores. Out of 10 binding complexes generated for each miRNA-mRNA duplex docked with AGO protein, the highest-scoring complex was chosen as the best binding mode. However, a good dock score in all cases of docking between the selected miRNAs (mir-145, mir-126, let-7a, and mir-196b) and AGO protein implicated a strong interaction between them at the molecular level (Supplementary Table 5).

\section{Study of interaction between miRNAs and AGO protein at the molecular level}

Strong hydrophobic amino acid residues and amino acids with aromatic rings, which are relatively hydrophobic in nature, generally take part in hydrophobic interactions and contribute towards the stability in binding moieties while the protein interacts with macromolecules. From the inspection of the close amino acid residues of AGO protein surrounding the miRNAs (mir-145, mir-126, let-7a, mir-196b.1, and mir-196b.2), some strongly hydrophobic amino acids, like LEU 652, LEU 267, ALA 644, and ALA 648 in mir-145; LEU 267, LEU 277, LEU 279, LEU 281, ALA 278, ALA 648, and VAL 606 in mir-126; LEU 267, LEU 279, LEU 281, and ALA 278in let-7a; ALA 111, VAL 58, and LEU 132 in mir-196b.1; and VAL 549, VAL 152, and ALA 170 in mir-196b.2, were observed within a distance of $3.5 \AA$ (Table 1 , Fig. 2A-2E). Similarly, amino acids, like TYR 642, PHE 649, and PHE 647 in mir-145; PHE 649 in mir-126; and TYR 171 and TYR 135 in mir-196b.2, with aromatic rings were also found in the binding pocket of miRNAs (Table 1, Fig. 2A-2E) within a distance of $3.5 \AA$. The study of hydrogen bonding patterns between atoms is another major parameter to study the stability of interactions between bio-molecules at their atomic level. Some amino acids, like ARG 615, ARG 651, HIS 445, and PHE 649 in mir-145; SER 280, HIS 607, and THR 613 in mir-126; SER 280, ARG 608, GLY 612, LEU 281, and

Table 1. Amino acid residues of Argonaute protein participating in the interaction with mir-145, mir-126, let-7a, mir-196b.1, and mir-196b.2 within a distance of $3.5 \AA$

\begin{tabular}{|c|c|c|c|c|}
\hline \multirow{2}{*}{ miRNA } & \multicolumn{4}{|c|}{ Amino acid residues } \\
\hline & Residue & Hydrophobic interactions ${ }^{a}$ & With aromatic rings ${ }^{b}$ & Hydrogen bonding ${ }^{c}$ \\
\hline mir-145 & $\begin{array}{l}\text { ARG 661, LYS 575, HIS } \\
657, \text { PRO 650, ASN 449, } \\
\text { GLU } 442\end{array}$ & $\begin{array}{l}\text { LEU 652, LEU 267, ALA } \\
\text { 644, ALA } 648\end{array}$ & $\begin{array}{l}\text { TYR 642, PHE 649, PHE } \\
647\end{array}$ & $\begin{array}{l}\text { ARG 615, ARG 651, HIS } \\
445\end{array}$ \\
\hline $\operatorname{mir}-126$ & $\begin{array}{l}\text { ASP 269, ASP 590, GLY } \\
\text { 612, ARG 611, ARG 608, } \\
\text { PRO } 614\end{array}$ & $\begin{array}{l}\text { LEU 267, LEU 277, LEU } \\
\text { 279, LEU 281, ALA 278, } \\
\text { ALA 648, VAL } 606\end{array}$ & PHE 649 & SER 280, THR 613, HIS 607 \\
\hline let-7a & $\begin{array}{l}\text { ARG 192, ARG 611, ARG } \\
\text { 615, THR 266, PRO 614, } \\
\text { HIS } 607\end{array}$ & $\begin{array}{l}\text { LEU 267, LEU 279, LEU } \\
\text { 281, ALA } 278\end{array}$ & - & $\begin{array}{l}\text { ARG 608, THR 613, SER } \\
\text { 280, GLY } 612\end{array}$ \\
\hline mir-196b.1 & $\begin{array}{l}\text { GLN 551, GLN 118, PRO } \\
\text { 103, GLY 104, GLY 131, } \\
\text { SER 107, ARG 114, GLU } \\
130\end{array}$ & ALA 111, VAL 58, LEU 132 & - & ARG 51, ARG 59 \\
\hline mir-196b.2 & $\begin{array}{l}\text { ARG 200, ARG 661, ARG } \\
\text { 548, ARG 574, ASP 246, } \\
\text { ASP 168, PRO 247, LYS } \\
\text { 248, LYS 575, GLY 547, } \\
\text { GLY } 579\end{array}$ & $\begin{array}{l}\text { VAL 549, VAL 152, ALA } \\
170\end{array}$ & TYR 171, TYR 135 & $\begin{array}{l}\text { ARG 580, GLY 577, SER } \\
576\end{array}$ \\
\hline
\end{tabular}

$\overline{{ }^{a} A m i n o ~ a c i d ~ r e s i d u e s ~ i n v o l v e d ~ i n ~ h y d r o p h o b i c ~ i n t e r a c t i o n s ; ~}{ }^{b}$ Amino acid residues with aromatic rings; ${ }^{\mathrm{C}} \mathrm{Amino}$ acid residues participating in hydrogen bonding. 

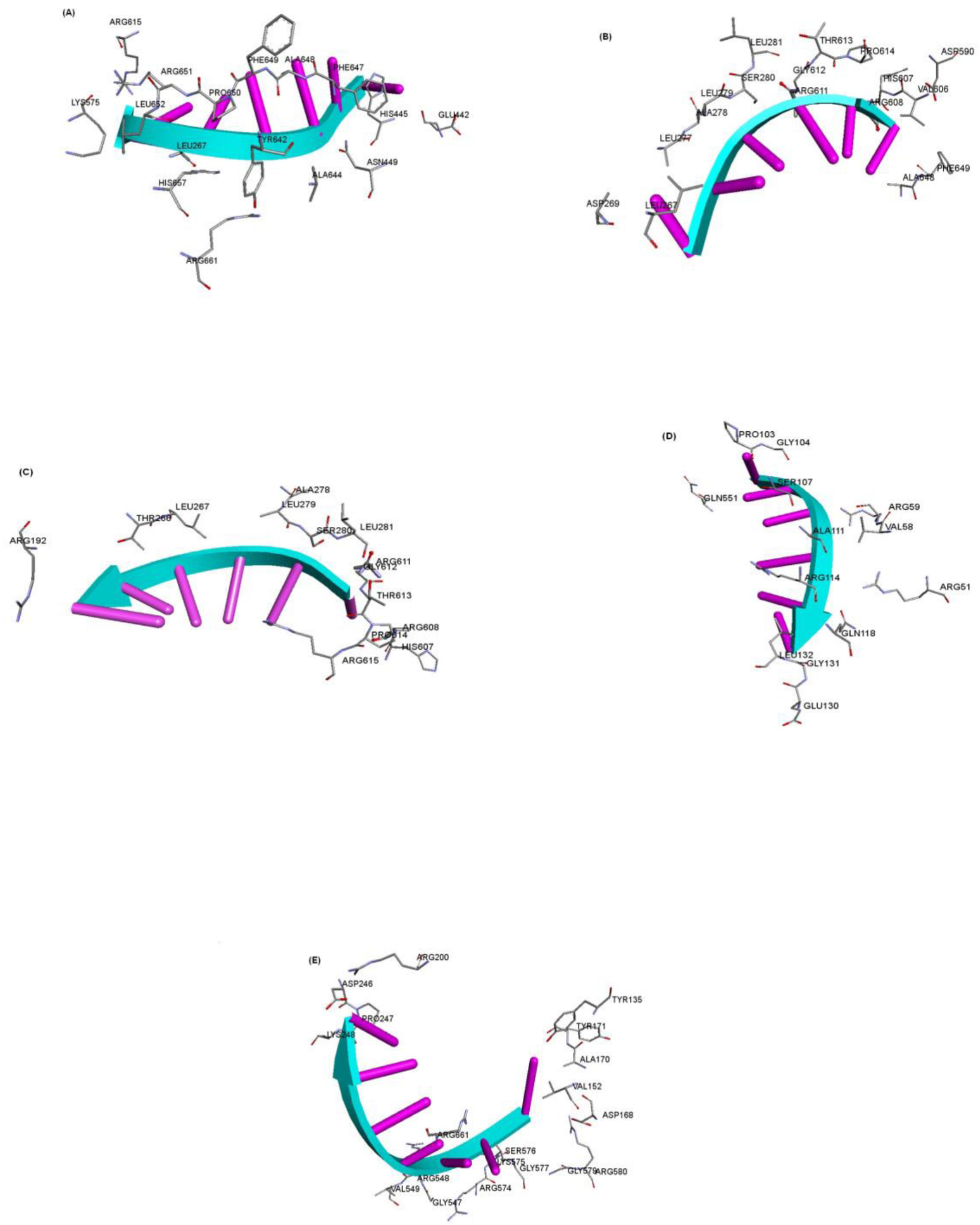

Fig. 2. Amino acid residues of Argonaute protein participating in the interaction with mir-145, mir-126, let-7a, mir-196b.1, and mir-196b.2 within distance of $3.5 \AA$ are reported in section (A), (B), (C), (D), and (E), respectively. 
RP Das, et al. Molecular Interaction of miRNAs and Target Gene

Table 2. Amino acid residues of Argonaute protein participating in hydrogen bonding with miRNAs within a distance of $3.0 \AA$

\begin{tabular}{|c|c|c|c|}
\hline miRNA & Residue & Atom & Distance $(\AA)$ \\
\hline \multirow[t]{5}{*}{ mir-145 } & ARG 615 & $\mathrm{NH} 1 \cdots \mathrm{O} 4$ (U17) & 2.47922 \\
\hline & ARG 615 & NH1...O2 (U17) & 2.10917 \\
\hline & ARG 651 & $\mathrm{NH} 2 \cdots \mathrm{O} 2(\mathrm{U} 16)$ & 2.45241 \\
\hline & HIS 445 & NE2 $\cdots H 42$ (C11) & 2.37822 \\
\hline & PHE 649 & O...H22 (U15) & 1.97722 \\
\hline \multirow[t]{4}{*}{$\operatorname{mir}-126$} & SER 280 & OG $\cdots$. O3 (U11) & 2.40718 \\
\hline & SER 280 & OG…O5 (A12) & 2.09735 \\
\hline & HIS 607 & $\mathrm{~N} \cdots . \mathrm{O} 2(\mathrm{C} 13)$ & 2.45673 \\
\hline & THR 613 & $\mathrm{~N} \cdots . \mathrm{O} 2(\mathrm{~A} 12)$ & 2.35176 \\
\hline \multirow[t]{5}{*}{ let-7a } & SER 280 & N...OP2 (G11) & 1.84591 \\
\hline & ARG 608 & N*N7 (G9) & 2.06803 \\
\hline & GLY 612 & $\mathrm{O} \cdots \mathrm{HO} 2$ (G9) & 1.45138 \\
\hline & LEU 281 & OOP1 (A10) & 1.83936 \\
\hline & THR 613 & 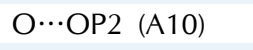 & 2.30494 \\
\hline \multirow[t]{4}{*}{ mir-196b.1 } & ARG 51 & NH1 $\cdots$ OP1 (G13) & 2.9226 \\
\hline & ARG51 & $\mathrm{NH} 2 \cdots \mathrm{OP} 1 \quad$ (G13) & 2.0137 \\
\hline & ARG 59 & N....OP1 (U11) & 2.90177 \\
\hline & ARG 59 & $\mathrm{NH} 2 \cdots \mathrm{OP} 1 \quad$ (U11) & 3.07531 \\
\hline \multirow[t]{4}{*}{ mir-196b.2 } & SER 576 & $\mathrm{~N} \cdots . . \mathrm{O} 2(\mathrm{G} 11)$ & 2.00398 \\
\hline & GLY 577 & N*..O4 (G11) & 2.40617 \\
\hline & ARG 580 & NE $\cdots .$. OP2 (A10) & 2.07775 \\
\hline & ARG 615 & $\mathrm{NH} 1 \cdots \mathrm{O} 3$ (A10) & 2.40811 \\
\hline
\end{tabular}

THR 613 in let-7a; ARG 51 and ARG 59 in mir-196b.1; and SER 576, GLY 577, ARG 580, and ARG 615 in mir-196b.2 (Tables 1 and 2, Fig. 3A-3E), were observed to participate in hydrogen bonding with miRNAs within the binding pocket.

\section{Discussion}

The current study focused on in silico verification of miRNAs for targeting the HOXA9 gene, associated in AML, and the molecular interaction of catalytic AGO protein with these miRNAs to study the gene silencing mechanism computationally. As the literature has reported [12, 13], four mature miRNAs - namely, miR-145, mir-126, let-7a, and mir-196b - are found to have target sites in the HOXA9 gene and were hence taken for further study. The binding sites in HOXA9 were predicted for these miRNAs using miRTarBase (Supplementary Tables 1 and 3), which was followed by the secondary structure prediction and miRNA-mRNA duplex formation. Two binding sites were predicted for mir-196b in the HOXA9 gene (Supplementary Table 1), denoted mir-196b.1 and mir-196b.2, respectively; so, two duplexes are formed between mir-196b and mRNA of the HOXA9 gene (Supplementary Table 3 ). The results suggested a strong and energetically favorable binding mode between all miRNA-mRNA duplexes (Supplementary Table 4), which is also supported by the reported interaction between them (Fig. 1). The good MFE score $(-5.90 \mathrm{kcal} / \mathrm{mol})$ also reflected that mir-145 had the highest binding affinity for the HOXA9 gene (Supplementary Table 4) among all miRNAs. Furthermore, to study the molecular interaction between AGO protein and miRNA-mRNA duplexes (miR-145, mir-126, let-7a, mir-196b.1, and mir-196b.2), molecular docking was carried out. The presence of strong hydrophobic amino acid residues (LEU 652, LEU 267, ALA 644, and ALA 648 in mir-145; LEU 267, LEU 277, LEU 279, LEU 281, ALA 278, ALA 648, and VAL 606 in mir-126; LEU 267, LEU 279, LEU 281, and ALA 278 in let-7a; ALA 111, VAL 58, and LEU 132 in mir-196b.1; and VAL 549, VAL 152, and ALA 170 in mir-196b.2) within a distance of $3.5 \AA$ in the binding pocket of miRNAs implicates strong binding interactions at the molecular level between AGO protein and all chosen miRNAs (Table 1, Fig. 2A-2E). Also, amino acids with aromatic rings (TYR 642, PHE 649, and PHE 647 in mir-145; PHE 649 in mir-126; and TYR 171 and TYR 135 in mir-196b.2) were also observed within a distance of $3.5 \AA$, which supports the binding interaction (Table 1, Fig. 2A-2E) between AGO protein and miRNAs. The amino acid residues participated in hydrogen bonding (Table 2, Fig. 3A-3E) with miRNAs (miR-145, mir-126, let-7a, mir-196b.1, and mir-196b.2) within a distance of $3.0 \AA$, showing strong evidence of binding and a molecular interaction between AGO protein and all chosen miRNAs. The current investigation proved that mir-145, mir-126, let-7a, mir-196b.1, and mir-196b.2 have strong binding affinity 

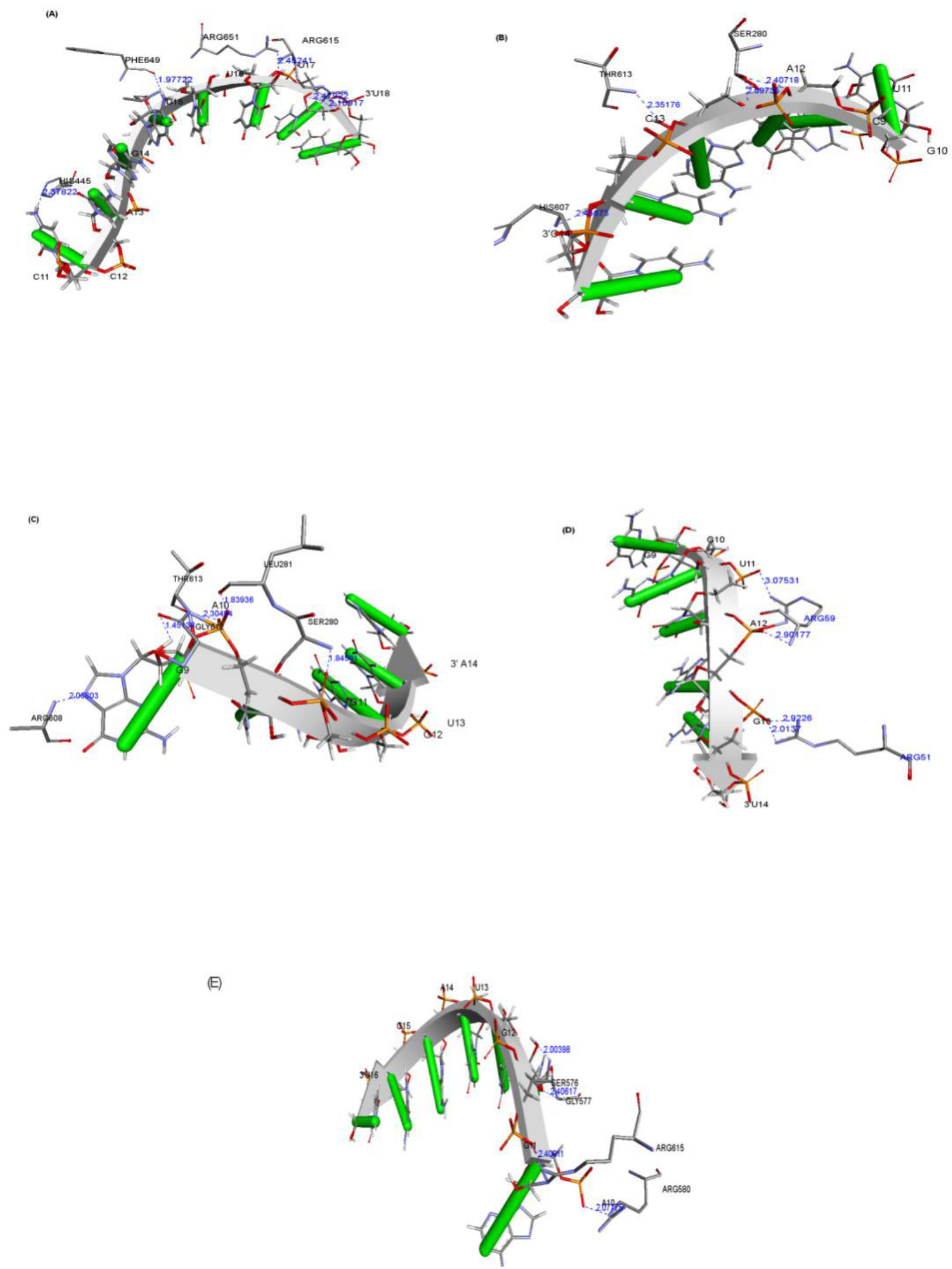

Fig. 3. Amino acid residues of Argonaute protein participating in hydrogen bonding interaction with mir-145, mir-126, let-7a, mir-196b.1, and mir-196b.2 within distance of $3.0 \AA$ are reported in section (A), (B), (C), (D), and (E), respectively. 
for the HOXA9 gene and also throws light on AGO protein-assisted molecular interactions with miRNA-mRNA duplex structures. This report would be helpful in understanding miRNA-based gene silencing mechanisms for the HOXA9 gene in AML disease. Furthermore, a molecular dynamic simulation approach might be implemented to study the dynamic behavior of these interactions, which would help us better understand miRNA-based gene silencing mechanisms in AML computationally. Once validated experimentally, this methodology would further help us to design suitable miRNAs against respective genes rationally.

\section{Supplementary materials}

Supplementary data including five tables can be found with this article online at http://www.genominfo.org/src/ sm/gni-13-45-s001.pdf.

\section{Acknowledgments}

The authors are thankful to the Department of Biotechnology, Govt. of India, for providing the BIF Centre facility to carry out the current research work. Also, the authors are grateful to the authorities of the Department of Bioinformatics, Centre for Post Graduate Studies, Orissa University of Agriculture \& Technology, Bhubaneswar, Odisha, India, for their constant encouragement and allowing this research work to be accomplished.

\section{References}

1. Didiano D, Hobert O. Molecular architecture of a miRNAregulated 3' UTR. RNA 2008;14:1297-1317.

2. Gu W, Xu Y, Xie X, Wang T, Ko JH, Zhou T. The role of RNA structure at $5^{\prime}$ untranslated region in microRNA-mediated gene regulation. RNA 2014;20:1369-1375.

3. Rajewsky N. MicroRNA target predictions in animals. Nat Genet 2006;38 Suppl:S8-S13.
4. Hutvagner G, Simard MJ. Argonaute proteins: key players in RNA silencing. Nat Rev Mol Cell Biol 2008;9:22-32.

5. Farazi TA, Ten Hoeve JJ, Brown M, Mihailovic A, Horlings HM, van de Vijver MJ, et al. Identification of distinct miRNA target regulation between breast cancer molecular subtypes using AGO2-PAR-CLIP and patient datasets. Genome Biol 2014; 15:R9.

6. Betel D, Koppal A, Agius P, Sander C, Leslie C. Comprehensive modeling of microRNA targets predicts functional non-conserved and non-canonical sites. Genome Biol 2010; 11:R90.

7. Macfarlane LA, Murphy PR. MicroRNA: biogenesis, function and role in cancer. Curr Genomics 2010;11:537-561.

8. Reinhart BJ, Slack FJ, Basson M, Pasquinelli AE, Bettinger JC, Rougvie AE, et al. The 21-nucleotide let-7 RNA regulates developmental timing in Caenorhabditis elegans. Nature 2000; 403:901-906.

9. Eulalio A, Huntzinger E, Izaurralde E. Getting to the root of miRNA-mediated gene silencing. Cell 2008;132:9-14.

10. Ahmad J, Hasnain SE, Siddiqui MA, Ahamed M, Musarrat J, Al-Khedhairy AA. MicroRNA in carcinogenesis and cancer diagnostics: a new paradigm. Indian J Med Res 2013;137: 680-694.

11. Wang Y, Li Y, Ma Z, Yang W, Ai C. Mechanism of microRNAtarget interaction: molecular dynamics simulations and thermodynamics analysis. PLoS Comput Biol 2010;6:e1000866.

12. Shen WF, Hu YL, Uttarwar L, Passegue E, Largman C. MicroRNA-126 regulates HOXA9 by binding to the homeobox. Mol Cell Biol 2008;28:4609-4619.

13. Li Z, Huang H, Chen P, He M, Li Y, Arnovitz S, et al. miR-196b directly targets both HOXA9/MEIS1 oncogenes and FAS tumour suppressor in MLL-rearranged leukaemia. Nat Commun 2012;3:688.

14. Thorsteinsdottir U, Kroon E, Jerome L, Blasi F, Sauvageau G. Defining roles for HOX and MEIS1 genes in induction of acute myeloid leukemia. Mol Cell Biol 2001;21:224-234.

15. Alharbi RA, Pettengell R, Pandha HS, Morgan R. The role of HOX genes in normal hematopoiesis and acute leukemia. Leukemia 2013;27:1000-1008.

16. Schneidman-Duhovny D, Inbar Y, Nussinov R, Wolfson HJ. PatchDock and SymmDock: servers for rigid and symmetric docking. Nucleic Acids Res 2005;33:W363-W367. 